\title{
Ultraschallkurse in Ungarn
}

Immer größerer Beliebtheit erfreuen sich die Kurse im ,Csipkerozsa-Park-Resort" in Süd-Ungarn, welche alle SGUM-konform sind und durch Schweizerische Kursleiter in deutscher Sprache abgehalten werden. Die Verbindung von intensivem und individuell optimiertem Lernen in kleinen Gruppen einerseits und das Kennenlernen von lokaler Kultur und Gastronomie andererseits, bietet eine nützliche und interessante Abwechslung zum Klinik- oder Praxis-Alltag. Zunehmend werden auch Begleitpersonen und ganze Familien mitgenommen, welche vom großzügigen Freizeitangebot des Resorts Gebrauch machen können. Zudem erlauben die lokalen Preise äußerst günstiges Shopping.

Im Jahr 2015 sind bis jetzt folgende Kurse geplant (weitere werden noch organisiert):
- Monatlich 1-2 Supervisions-Kurse ( 3 Tage oder 7 Tage) mit individuellem Training in Kleinstgruppen, am Bewegungsapparat, den Weichteilen und auch am Abdomen.

- Grundkurs Sonografie des Bewegungsapparats.

- Refresherkurs Abdomen für Pädiater.

- Workshop/Expertenkurs für Sonografie der Inguinalregion.

- Workshop/Expertenkurs für Sonografie in der Sportmedizin.

Alle Einzelheiten der Kurse, zu welchen auch Kolleginnen und Kollegen aus dem Ausland willkommen sind, können unter www.ungarnkurse.ch eingesehen werden.

\section{Beat Dubs}

\begin{tabular}{|c|c|c|c|}
\hline Kursname & Beginn/ Ende & Kursort & Kursleiter \\
\hline $\begin{array}{l}\text { Supervisions- und Intensiv-Trainingskurs } \\
1 / 2015 \text { Sonografie des Abdomens, der } \\
\text { Weichteile und des Bewegungsapparates }\end{array}$ & $\begin{array}{l}15.01 .2015- \\
18.01 .2015\end{array}$ & $\begin{array}{l}\text { Csòlyospàlos } \\
\text { (Ungarn) }\end{array}$ & $\begin{array}{l}\text { Dr. med. Beat } \\
\text { Dubs }\end{array}$ \\
\hline $\begin{array}{l}\text { Supervisions- und Intensiv-Trainingskurs } \\
\text { 2/2015 Sonografie des Abdomens, der } \\
\text { Weichteile und des Bewegungsapparates }\end{array}$ & $\begin{array}{l}05.02 .2015- \\
08.02 .2015\end{array}$ & $\begin{array}{l}\text { Csòlyospàlos } \\
\text { (Ungarn) }\end{array}$ & $\begin{array}{l}\text { Dr. med. Beat } \\
\text { Dubs }\end{array}$ \\
\hline $\begin{array}{l}\text { Supervisions- und Intensiv-Trainingskurs } \\
\text { 3/2015 Sonografie des Abdomens, der } \\
\text { Weichteile und des Bewegungsapparates }\end{array}$ & $\begin{array}{l}19.02 .2015- \\
22.02 .2015\end{array}$ & $\begin{array}{l}\text { Csòlyospàlos } \\
\text { (Ungarn) }\end{array}$ & $\begin{array}{l}\text { Dr. med. Beat } \\
\text { Dubs }\end{array}$ \\
\hline $\begin{array}{l}\text { Wochen-Supervisions-Trainingskurs 4/2015 } \\
\text { Sonografie der Weichteile und des Bewe- } \\
\text { gungsapparates }\end{array}$ & $\begin{array}{l}01.03 .2015- \\
08.03 .2015\end{array}$ & $\begin{array}{l}\text { Csòlyospàlos } \\
\text { (Ungarn) }\end{array}$ & $\begin{array}{l}\text { Dr. med. Beat } \\
\text { Dubs }\end{array}$ \\
\hline $\begin{array}{l}\text { Supervisions- und Intensiv-Trainingskurs } \\
\text { 5/2015 Sonografie des Abdomens, der } \\
\text { Weichteile und des Bewegungsapparates }\end{array}$ & $\begin{array}{l}19.03 .2015- \\
22.03 .2015\end{array}$ & $\begin{array}{l}\text { Csòlyospàlos } \\
\text { (Ungarn) }\end{array}$ & $\begin{array}{l}\text { Dr. med. Beat } \\
\text { Dubs }\end{array}$ \\
\hline Sonografie in der Sportmedizin & $\begin{array}{l}16.04 .2015- \\
19.04 .2015\end{array}$ & $\begin{array}{l}\text { Csòlyospàlos } \\
\text { (Ungarn) }\end{array}$ & $\begin{array}{l}\text { Dr. med. Andre } \\
\text { Dietschi }\end{array}$ \\
\hline $\begin{array}{l}\text { Grundkurs Hüftsonografie } 2015 \text { (unga- } \\
\text { risch) }\end{array}$ & $\begin{array}{l}23.04 .2015- \\
24.04 .2015\end{array}$ & $\begin{array}{l}\text { Csòlyospàlos } \\
\text { (Ungarn) }\end{array}$ & $\begin{array}{l}\text { Dr. med. Ger- } \\
\text { trud Remsei }\end{array}$ \\
\hline $\begin{array}{l}\text { Aufbaukurs Hüftsonografie } 2015 \text { (unga- } \\
\text { risch) }\end{array}$ & $\begin{array}{l}24.04 .2015- \\
25.04 .2015\end{array}$ & $\begin{array}{l}\text { Csòlyospàlos } \\
\text { (Ungarn) }\end{array}$ & $\begin{array}{l}\text { Dr. med. Ger- } \\
\text { trud Remsei }\end{array}$ \\
\hline $\begin{array}{l}\text { Supervisions- und Intensiv-Trainingskurs } \\
6 / 2015 \text { Sonografie des Abdomens, der } \\
\text { Weichteile und des Bewegungsapparates }\end{array}$ & $\begin{array}{l}07.05 .2015- \\
10.05 .2015\end{array}$ & $\begin{array}{l}\text { Csòlyospàlos } \\
\text { (Ungarn) }\end{array}$ & $\begin{array}{l}\text { Dr. med. Beat } \\
\text { Dubs }\end{array}$ \\
\hline $\begin{array}{l}\text { Supervisions- und Intensiv-Trainingskurs } \\
7 / 2015 \text { Sonografie des Abdomens, der } \\
\text { Weichteile und des Bewegungsapparates }\end{array}$ & $\begin{array}{l}28.05 .2015- \\
31.05 .2015\end{array}$ & $\begin{array}{l}\text { Csòlyospàlos } \\
\text { (Ungarn) }\end{array}$ & $\begin{array}{l}\text { Dr. med. Beat } \\
\text { Dubs }\end{array}$ \\
\hline $\begin{array}{l}\text { Supervisions- und Intensiv-Trainingskurs } \\
8 / 2015 \text { Sonografie des Abdomens, der } \\
\text { Weichteile und des Bewegungsapparates }\end{array}$ & $\begin{array}{l}11.06 .2015- \\
14.06 .2015\end{array}$ & $\begin{array}{l}\text { Csòlyospàlos } \\
\text { (Ungarn) }\end{array}$ & $\begin{array}{l}\text { Dr. med. Beat } \\
\text { Dubs }\end{array}$ \\
\hline $\begin{array}{l}\text { Grundkurs Sonografie des Bewegungsap- } \\
\text { parates } 2015\end{array}$ & $\begin{array}{l}25.06 .2015- \\
28.06 .2015\end{array}$ & $\begin{array}{l}\text { Csòlyospàlos } \\
\text { (Ungarn) }\end{array}$ & $\begin{array}{l}\text { Dr. med. Beat } \\
\text { Dubs }\end{array}$ \\
\hline $\begin{array}{l}\text { Wochen-Supervisions-Trainingskurs 9/2015 } \\
\text { Sonografie der Weichteile und des Bewe- } \\
\text { gungsapparates }\end{array}$ & $\begin{array}{l}05.07 .2015- \\
12.07 .2015\end{array}$ & $\begin{array}{l}\text { Csòlyospàlos } \\
\text { (Ungarn) }\end{array}$ & $\begin{array}{l}\text { Dr. med. Beat } \\
\text { Dubs }\end{array}$ \\
\hline
\end{tabular}

